\title{
Correction to: Exploring the Genetic Association of the ABAT Gene with Alzheimer's Disease
}

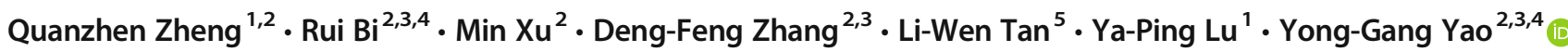

Published online: 22 January 2021

(C) Springer Science+Business Media, LLC, part of Springer Nature 2021

\section{Correction to: Mol Neurobiol.} https://doi.org/10.1007/s12035-020-02271-z

The original version of this article unfortunately contained some mistakes introduced by the Publisher during the proofreading stage.

Assignment of affiliation number to "Yong-Gang Yao" IS incorrect. Dr. Yao is not affiliated to Mental Health Institute of the Second Xiangya Hospital but should be with Center for Excellence in Brain Science and Intelligence Technology, Chinese Academy of Sciences.

"Yong-Gang Yao $\mathrm{Ya}^{2,3,5 "}$ should be "Yong-Gang Yao ${ }^{2,3,4,}$. The original paper has been corrected.

Publisher's Note Springer Nature remains neutral with regard to jurisdictional claims in published maps and institutional affiliations.

The online version of the original article can be found at https://doi.org/ 10.1007/s12035-020-02271-z

Yong-Gang Yao

yaoyg@mail.kiz.ac.cn

1 College of Life Sciences, Anhui Normal University, Wuhu 241002, Anhui, China

2 Key Laboratory of Animal Models and Human Disease Mechanisms of the Chinese Academy of Sciences \& Yunnan Province, and KIZ/ CUHK Joint Laboratory of Bioresources and Molecular Research in Common Diseases, Kunming Institute of Zoology, Chinese Academy of Sciences, Kunming 650204, Yunnan, China

3 Kunming College of Life Science, University of Chinese Academy of Sciences, Kunming 650204, China

4 Center for Excellence in Brain Science and Intelligence Technology, Chinese Academy of Sciences, Shanghai 200031, China

5 Mental Health Institute of the Second Xiangya Hospital, Central South University, Changsha 410011, China 